this time. Overall, a vast majority of respondents found the simulation to be a worthwhile exercise that increased knowledge and preparedness. An overwhelming majority also reported that the simulation was relevant to practice, of high quality, and a useful way to teach about major incidents.

Discussion: Our surprise major incident simulation was a fun and effective way to raise awareness and increase knowledge in prehospital and emergency department clinicians about MCI response. This approach to simulation can be easily replicated at relatively low cost and is, therefore, a useful solution to training a group of multidisciplinary health professionals outside of the workplace.

Prehosp. Disaster Med. 2019;34(Suppl. 1):s18-s19

doi:10.1017/S1049023X19000554

\section{Training Emergency Department Charge Nurses Through} Tabletop Exercises

Ms. Beth Weeks

University Of Colorado Hospital - UCHealth, Denver, United States

Introduction: In a disaster or mass casualty incident, the Emergency Department (ED) charge nurse is thrust into an expanded leadership role, expected to not only manage the department but also organize a disaster response. Hospital emergency preparedness training programs typically focus on high-level leadership, while frontline decision-making staff get experience only through online training and infrequent full-scale exercises. Financial and time limitations of full-scale exercises have been identified as major barriers to frontline training.

Aim: To discuss a cost-effective approach to training ED charge nurses and informal leaders in disaster response.

Methods: A formal training program was implemented in the ED. All permanent and relief charge nurses are required to attend one four-hour Hospital ICS course within their first year in their position, as well as participate in a minimum of one two-hour ED-based tabletop exercise per year. The tabletop exercises are offered bimonthly, covering various mass casualty scenarios such as apartment complex fires, riots, and a tornado strike. Full-scale exercises involving the ED occur annually.

Results: ED permanent and relief charge nurses expressed increased skills and knowledge in areas such as initiation of disaster processes, implementation of hospital incident command, and familiarization with protocols and available resources. Furthermore, ED charge nurses have demonstrated strong leadership, decision-making, and improved response to actual mass casualty incidents since implementing ICS training and tabletop exercises.

Discussion: Limitations of relying on full-scale disaster exercises to provide experience to frontline leaders can be overcome by the inclusion of ICS training and tabletop exercises for ED charge nurses in a hospital training and exercise plan. Implementing a structured training program for ED charge nurses focusing on leadership in mass casualty incidents is one step to building a more resilient and prepared ED, hospital, and community.

Prehosp. Disaster Med. 2019;34(Suppl. 1):s19

doi:10.1017/S1049023X19000566

\section{Use of Moulage in Multi-Disciplinary Mass Casualty Incident (MCI) Training: Cost-Effective Tool or an Expectation?}

Dr. Sasha Ribter ${ }^{1}$, Dr. Veronica Coppersmith ${ }^{2}$

1. Ohio Valley Medical Center, Wheeling, United States

2. St. Luke's University, University Health Network, Bethlehem, United States

Introduction: A 2018 poll by the American College of Emergency Physicians shows 93\% of surveyed doctors believe their emergency department is not fully prepared for patient surge capacity in the event of a natural or man-made disaster. While an emergency disaster plan is activated during any incident where resources are overwhelmed, many US emergency physicians today think of a mass casualty incident (MCI) as the inciting event. To better prepare our communities, an MCI simulation took place in Chicago 2018 with participation from local and federal representatives. Included were Chicago fire, police, and emergency medical services agencies, emergency medicine physicians, resident participants, and medical student volunteer victims.

Aim: The study's aim was to determine whether resource intensive moulage was an expected component or a beneficial adjunct, if moulage-based training would improve physician preparedness, and if such a training would increase the likelihood of future involvement in local disaster preparations. Analysis was performed on pre- and post-training surveys completed by participants. By reviewing the benefits versus cost, future MCI simulation planners can efficiently use their funds to achieve training goals.

Methods: Thirty-two emergency medicine physicians were surveyed before and after a five-hour training session on October 20, 2018, which included 89 moulage victims. Twenty-four afterevent surveys were completed. All completed surveys were utilized in data analysis.

Results: Of polled participants, a $68 \%$ improvement in general preparedness was achieved. While only $19 \%$ of participants cited current involvement in their facility's disaster planning in pre-event survey, the likelihood of involvement after training was 8.2/10. Overall, the importance of moulage an essential component to such trainings remained constant.

Discussion: Moulage is an expected and crucial element to MCI training and should be incorporated as extensively as resources allow. MCI trainings improve physician preparedness and potentially increase physician involvement in disaster planning at home institutions.

Prehosp. Disaster Med. 2019;34(Suppl. 1):s19

doi:10.1017/S1049023X19000578 